\title{
Regularity of Solutions of Degenerate Parabolic Non-linear Equations and Removability of Solutions
}

Gadjiev TS*, Yangaliyeva A and Zulfalieva G

Institute of Mathematics and Mechanics of NAS of Azerbaijan, AZ1141 Baku, Azerbaijan

\author{
Abstract \\ In this paper we prove regularity of solutions of degenerate parabolic nonlinear equations. We also the proof of \\ a removability theorem for solutions to degenerate parabolic nonlinear equations.
}

Keywords: Degenerate; Nonlinear parabolic equations; Regularity; Removability

\section{Introduction}

Let we are considered in cylindrical domains $\mathrm{Q}_{\mathrm{T}=} \Omega \times(0, \mathrm{~T})$, where $\Omega \subset \mathrm{R}^{\mathrm{n}}, \mathrm{n} \geq 2$ is a bounded Lipschitz domain, $\mathrm{T}>0$, degenerate nonlinear parabolic equations

$$
\begin{aligned}
& \mathrm{u}_{\mathrm{t}}-\operatorname{div}\left(\omega(\mathrm{x})|\mathrm{Du}|^{\mathrm{p}-2} \mathrm{Du}\right)=0 \\
& \left.\left.\mathrm{u}\right|_{\Gamma(\mathrm{QT}}\right)=\mathrm{h},
\end{aligned}
$$

where $\Gamma\left(\mathrm{Q}_{\mathrm{T}}\right)=\left(\Omega^{-} \times\{0\}\right) \cup(\partial \Omega \times[0, \mathrm{~T}])$ denote the parabolic boundary of $\mathrm{Q}_{\mathrm{T}}, \mathrm{h}: \mathrm{Q}_{\mathrm{T}} \rightarrow \mathrm{R}$ continuous function, $\omega(\mathrm{x})$-Makenxhoupt weight function [1].

To regularity of solutions to the degenerate parabolic non-linear operator introduced by DiBenedetto et al. [2,3]. Let $C_{w}^{\alpha}\left(Q_{T}\right)$ weighted space, where norm following:

$$
\|f\|_{C_{w(x)}^{\alpha}\left(Q_{T}\right)}=\sup _{z_{1}, z_{2} \in Q r} \frac{\left|f\left(z_{1}\right) w\left(x_{1}\right)-f\left(z_{2}\right) w\left(x_{2}\right)\right|}{\left\|z_{1}-z_{2}\right\| \alpha}<\infty
$$

where the parabolic metric is defined as

$$
\left\|\left(x_{1}, t_{1}\right)-\left(x_{2} t_{2}\right)\right\|_{\alpha}=\max \left[\left|x_{1}-x_{2}\right|\right],\left|t_{1}-t_{2}\right| \frac{1}{[p-\alpha(p-2)]}, 0<\alpha<1
$$

\section{Main Results}

We are now ready to state our result which concerns regularity for solutions to the problem (1.1), (1.2).

\section{Theorem 2.1}

Let's consider problem (1.1), (1.2)and let $\mathrm{u}(\mathrm{x}, \mathrm{t})$ solve this problem. Let $\mathrm{Q}_{\mathrm{T}}^{0} \subset \mathrm{Q}_{\mathrm{T}}$ be a bounded space time cylinder such that (interior regularity)

$$
\begin{aligned}
& \mathrm{Q}_{\mathrm{T}}^{0} \cap \Gamma\left(\mathrm{Q}_{\mathrm{T}}\right)=\emptyset \text {. Then } u \in C_{w(\mathrm{x})}^{\alpha}\left(Q_{T}^{\prime}\right) \text { and } \\
& \|u(\mathrm{x}, \mathrm{t})\|_{u \in C_{w(x)}^{\alpha}\left(Q_{T}^{\prime}\right)} \leq c\left(\mathrm{n}, \mathrm{p}, \mathrm{w}(\mathrm{x}), \mathrm{Q}_{T}, \mathrm{Q}_{T}^{\prime},\left|h(\mathrm{x}) \in C_{w(\mathrm{x})}^{\alpha}(\Omega), \operatorname{osu}(\mathrm{x}, \mathrm{t})\right|\right)
\end{aligned}
$$

Theorem 2.1 concerns optimal interior regularity. We also establish optimal regularity up to initial state. In particular, in this case we prove $C_{w}^{\alpha}\left(Q_{T}\right)$ estimates on $\left.Q_{r}=\Omega \times(0, T)\right)$ for every $\Omega, \subset \Omega$. We doing remark that in this case $Q_{T}$ is not a compact subset of $\mathrm{Q}_{\mathrm{T}}$.

In this context hold following result [1-12].

\section{Theorem 2.2}

Let $\mathrm{u}(\mathrm{x}, \mathrm{t})$ solve problem (1.1), (1.2) and(Initial time regularity)

$$
\begin{aligned}
& h(\mathrm{x}) \in C_{w(\mathrm{x})}^{\alpha}(\Omega), \Omega^{\prime} \subset \Omega Q_{T}^{\prime}=\Omega^{\prime} X(0, \mathrm{~T}), u \in C_{w(\mathrm{x})}^{\alpha}\left(Q_{T}^{\prime}\right) \\
& \text { And } \\
& \|u(\mathrm{x}, \mathrm{t})\|_{u \in C_{w(\mathrm{x})}^{\alpha}\left(Q_{T}^{\prime}\right)} \leq c\left(\mathrm{n}, \mathrm{p}, \mathrm{w}(\mathrm{x}), \mathrm{Q}_{T}, \mathrm{Q}_{T}^{\prime}, h(\mathrm{x}) \in C_{w(\mathrm{x})}^{\alpha}(\Omega), \mathrm{osu}(\mathrm{x}, \mathrm{t}) \mid\right)
\end{aligned}
$$

We also can be is considered obstacle problem similarly to problem (1.1), (1.2). In the case of linear uniformly parabolic equations [4]. Optimal regularity problem of the solution is considered [5].

We are study weak solutions from $L^{P}\left(\mathrm{t}_{1} \mathrm{t}_{2}, \mathrm{~W}_{w(\mathrm{x})}^{1, p}\left(\mathrm{Q}_{T}\right)\right)$ space. In the space

$\mathrm{W}_{w(\mathrm{x})}^{1, p}(\Omega)$ the norm denote the space of equivalence classes of functions $f$ with distributional gradient $D f$, both of which are $\mathrm{p}^{\text {th }}$ power integral on $\mathrm{Q}_{\mathrm{T}}$. Let

$$
\|f\|_{\mathrm{w}_{w(x)}^{1, p}(\Omega)}=\|w(\mathrm{x}) \mathrm{f}(\mathrm{x})\|_{L P(\Omega)}+\|w(\mathrm{x})\||D f|_{L P(\Omega)}
$$

be the norm in $\mathrm{W}_{w(\mathrm{x})}^{1, p}(\Omega)$.

Given $\mathrm{t}_{1}<\mathrm{t}_{2}$ we denote by $L^{P}\left(\mathrm{t}_{1} \mathrm{t}_{2}, \mathrm{~W}_{w(x)}^{1, p}\left(\mathrm{Q}_{T}\right)\right)$ the space of functions such that for almost every $\mathrm{t}, \mathrm{t}_{1} \leq \mathrm{t} \leq \mathrm{t}_{2}$ the function

$$
\begin{aligned}
& \mathrm{x} \rightarrow \mathrm{u}(\mathrm{x}, \mathrm{t}) \text { belongs to } \mathrm{W}_{w(\mathrm{x})}^{1, p}(\Omega) \text { and }\|u\|_{L^{P}\left(\mathrm{t}_{1} \mathrm{t}_{2}, \mathrm{~W}_{w(\mathrm{x})}^{1, p}\left(\mathrm{Q}_{T}\right)\right)} \\
&=\left(\int_{t_{1}}^{t_{2}} \int_{\Omega}\left(\mathrm{w}(\mathrm{x})|u(\mathrm{x}, \mathrm{t})|^{p}+w(\mathrm{x})|D u(\mathrm{x}, \mathrm{t})|^{p} d x d t\right)^{\frac{1}{P}} \leq \infty\right.
\end{aligned}
$$

We say that a function $\mathrm{u}(\mathrm{x}, \mathrm{t})$ is a weak solution to (1.1), (1.2) in an open set

$\mathrm{Q}_{\mathrm{T}} \subset \mathrm{R}^{\mathrm{n}+1}$ if whenever $\mathrm{Q}_{\mathrm{T}}^{0} \Omega^{0} \times\left(\mathrm{t}_{1}, \mathrm{t}_{2}\right) \subset \mathrm{Q}_{\mathrm{T}}$ with $\Omega^{0} \subset \Omega \subset \mathrm{R}^{\mathrm{n}}$ and $\mathrm{t}_{1}<\mathrm{t}_{2}$ then $u \in L^{P}\left(\mathrm{t}_{1} \mathrm{t}_{2}, \mathrm{~W}_{w(\mathrm{x})}^{1, p}(\Omega)\right)$ and

$$
\int_{Q_{T}}\left(\mathrm{w}(\mathrm{x})|D u|^{p-2} D u D \varphi-u \varphi_{t}\right) d x d t=0
$$

for all nonnegative $\varphi \in C_{0}^{\infty}\left(Q_{T}^{\prime}\right)$.

*Corresponding author: Gadjiev TS, Institute of Mathematics and Mechanics of NAS of Azerbaijan, AZ1141 Baku, Azerbaijan, Tel: +994506728756; E-mail tgadjiev@mail.az

Received July 07, 2017; Accepted August 21, 2017; Published August 31, 2017

Citation: Gadjiev TS, Yangaliyeva A, Zulfalieva G (2017) Regularity of Solutions of Degenerate Parabolic Non-linear Equations and Removability of Solutions. J Appl Computat Math 6: 364. doi: 10.4172/2168-9679.1000364

Copyright: (C) 2017 Gadjiev TS, et al. This is an open-access article distributed under the terms of the Creative Commons Attribution License, which permits unrestricted use, distribution, and reproduction in any medium, provided the original author and source are credited. 
Using Theorem 2.1 we are able to establish sharp removability conditions for compact sets. We of cylinders introduced

$$
Q_{T}^{\lambda}\left(\mathrm{x}_{0}, \mathrm{t}_{0}\right)=\left\{(\mathrm{x}, \mathrm{t}) \in \mathrm{R}^{n+1}:\left|x_{0}-x\right|<r,\left|t_{0}-t\right|<\lambda^{2-p \tau p}\right\}
$$

And a concave modulus of continuity $\psi(\cdot)$. We let $\psi: R_{+} \rightarrow R_{+}$ be a concave modulus of continuity, i.e., concave non-decreasing function such that $\psi(1)=1$ and $\psi(0)=\lim _{r \rightarrow 0} \psi(r)=0$. We also define Hausdorff measure as follows. We let for fixed $\delta, 0<\delta<\mathrm{r}_{0}$ and $E \subset R^{n+1}, L(\delta, \psi(.) ; \mathrm{E})=\left\{Q_{r i}^{\psi(\mathrm{ri})}\left(\mathrm{x}_{\mathrm{i}}, \mathrm{t}_{\mathrm{i}}\right)\right\}$ be a family of cylinders such that $E \subset \cup Q_{r i}^{\psi(\mathrm{ri})}\left(\mathrm{x}_{\mathrm{i}}, \mathrm{t}_{\mathrm{i}}\right)$ and $0<\mathrm{r}_{\mathrm{i}}<\delta$ for $\mathrm{i}=1,2, .$.

Using this notation we let

$$
H^{\psi(.)}(\mathrm{E})=\lim _{\delta \downarrow 0} \inf _{L(\delta, \psi(.) ; \mathrm{E})}\left\{\sum r_{i}^{n} \psi\left(\mathrm{r}_{\mathrm{i}}\right): E \subset \cup Q_{r i}^{\psi(\mathrm{ri})}\left(\mathrm{x}_{\mathrm{i}}, \mathrm{t}_{\mathrm{i}}\right)\right\}
$$

where the indium is taken with respect to all possible coverings $\mathrm{L}(\delta, \psi(\cdot)$; E) of E.

\section{Theorem 2.3}

Let $\mathrm{Q}_{\mathrm{T}}$ be a cylindrical domain and let $\mathrm{E} \subset \mathrm{Q}_{\mathrm{T}}$ be a closed set. Let $\mathrm{u}(\mathrm{x}, \mathrm{t})$ is a weak solution to eqn. (1.1) in $\mathrm{Q}_{\mathrm{T}} \backslash \mathrm{E}$ and that $u(\mathrm{x}, \mathrm{t}) \in C_{w(\mathrm{x})}^{\alpha}\left(\mathrm{Q}_{T}\right)$

Assume also that $\mathrm{H}^{\psi(\cdot)}(\mathrm{E})=0$. Then the set $\mathrm{E}$ is removable, i.e., $\mathrm{u}(\mathrm{x}, \mathrm{t})$ can be extended to be a weak solution in $\mathrm{Q}_{\mathrm{T}}$.

Similarly result the fundamental work [6], under assumption Holder continuity of the solution can be found [7-12].

\section{Proof of theorem 2.1}

We assume $Q_{T} \subset Q_{T}$ such that $Q_{T}^{0} \cap \Gamma\left(Q_{T}\right)=\varnothing$.

We define function

$$
\underset{\Omega X[0, \infty]}{O S C} \bar{h}=\underset{\Omega X[0, T]}{O S C} h
$$

Then $\underset{\Omega X[0, \infty]}{\operatorname{osc}} \bar{h}=\underset{\Omega X[0, T]}{\operatorname{osc} h}$. Let ${ }^{-} u$ be the unique solution to

$$
\bar{u}_{t}-\operatorname{div}\left(\mathrm{w}(\mathrm{x})|D \bar{u}|^{p-2} D \bar{u}\right)=0 \text { in } \Omega \times(0, \infty)
$$

$u^{-}(x, t)=h^{-}(x, t)$ on $\Gamma(\Omega \times(0, \infty))$.

By the uniqueness ${ }^{-} u=u$ in $\Omega \times[0, T]$ and hence ${ }^{-} u$ is an extension of $u$. Let

\section{$R=\max \left\{1, \operatorname{diam} \Omega, T^{1 / 2}\right\}$. As clearly}

$$
T \leq(\psi(R))^{2-p} R^{p} \leq R^{2}
$$

Whenever $R \geq 1$. By maximum and minimum principle implies that

$$
\underset{Q r}{o s c u} \leq \underset{Q r}{\operatorname{oscu}} \leq \bar{c}(\Omega, T, \underset{Q r}{\operatorname{oscu}})
$$
let $R$ be

We may assume that $Q_{T, \gamma=}^{\prime} \Omega^{\prime} x(\tau, \mathrm{T})$, where $\Omega^{0} \subset \Omega$ and $\tau>0$. We

a number subject to the restrictions

$R \leq \operatorname{dist}\left(\Omega^{0}, \partial \Omega\right), \tau \geq R^{p} \max \{\mathrm{osch}, \psi(R), s \cdot R\}^{2-p}$.

$Q_{T}$

As so $\psi(1)=1$, we see that these conditions are satisfied if we take

$R \leq \operatorname{dist}\left(\Omega^{\prime}, \partial\left(\Omega^{\prime}, \partial \Omega\right)\right), \max \left\{T^{\frac{1}{P}}(\Omega, \mathrm{T}, \underset{Q T}{\operatorname{osch}})^{\frac{p-2}{p}}, \tau^{\frac{1}{P}}, \tau^{\frac{1}{r}}, \tau^{\frac{1}{2}}, S^{\frac{P-2}{P}}\right\}$ $\in Q_{T, \tau^{*}}^{0}$
Now we prove that the following holds whenever $z \in Q_{T}^{\prime}$

$$
\underset{Q_{T, r}^{c-\lambda \psi(\mathrm{r})}\left(\mathrm{z}_{0}\right) \cap Q \mathrm{Qr}}{\operatorname{OSc}} u \leq \underset{Q T}{\operatorname{oscu}}=\frac{\underset{Q T}{o s c u}}{\psi(\mathrm{r})} \leq \frac{\underset{Q T}{o s c u}}{\psi\left(\frac{R}{2}\right)} \leq 2 \lambda \psi(\mathrm{r})
$$

This completes the proof of Theorem 2.1.

\section{Proof of theorem 2.2}

After extending $u(x, t)$ as in the above we choose

$R=\operatorname{dist}\left(\Omega^{0}, \partial \Omega\right)$ and define

$$
\lambda=\max \left(\bar{c} / \psi(\mathrm{R}),|b|_{C_{w(x)}(\Omega)}, s . R / \psi(\mathrm{R})\right) \quad \text { where } \bar{c}=\bar{c}(\Omega, T, \underset{Q r}{\operatorname{oscu}})
$$

We let $Z=\Omega^{-0} \times(0)$ then

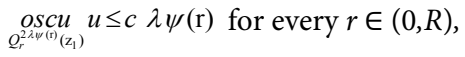

$Q \lambda \psi r(r)(z) \cap Q 0 T$.

Whenever $z \in Z$. Consider $z_{1} \in\left(Q_{T}^{\prime} \cap\left(\bar{\Omega}^{\prime} \times \mathrm{X}(0)\right)\right.$ and define $\bar{r}=\bar{r}\left(\mathrm{z}_{1}\right)=\sup \left[r \leq R: Q_{r}^{\lambda \psi(\mathrm{r})}\left(\mathrm{z}_{1}\right) \cap \mathrm{Z}=\theta\right]$ If $r>R / 2$, then

$\underset{Q_{r}^{2 \mu(r)}\left(\mathrm{z}_{1}\right)}{O S C u} u \quad \lambda \psi(\mathrm{r})$ for every $r \in(0, R)$.

In the final

$\lambda^{-}=\max \left\{4 \lambda \psi\left({ }^{-} r\right), s \cdot r / \psi^{-}\left({ }^{-} r\right)\right\} 4 \max \{\lambda, s R / \psi(R)\}=c \cdot \lambda$, implies that

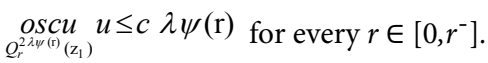

Whenever $z_{1} \in\left(Q_{T}^{\prime} \cap\left(\bar{\Omega}^{\prime} \mathrm{X}(0)\right)\right.$.

This completes the proof of Theorem 2.2.

\section{Proof of theorem 2.3}

Let $u(x, t)$ weakly solve of eqn. (1.1) in $Q_{T} \backslash E$ and assume that $u(\mathrm{x}, \mathrm{t}) \in \mathrm{C}_{w(\mathrm{x}) \text {,loc }}^{\alpha}\left(\mathrm{Q}_{T}\right)$ and $H^{\psi(\cdot)}(E)=0 . Q_{T}^{2} \subset Q_{T}^{1} \subset Q_{T}$ be arbitrary spacetime smooth cylinders. Our only need to prove the conclusion in $Q_{T}^{1}$ since the one of being a weak solution is a local property. By the assumption

$$
\begin{aligned}
& u(\mathrm{x}, \mathrm{t}) \in \mathrm{C}_{w(\mathrm{x}), \text { loc }}^{\alpha}\left(\mathrm{Q}_{T}\right) \text { there exists } M>0 \text { such that } \\
& \underset{Q_{T}^{\perp}}{\operatorname{osc}} u(\mathrm{x}, \mathrm{t}) \leq M \text { and } \underset{Q_{T}^{\mu-\psi(\mathrm{t})} \cap Q_{T}^{\prime}}{\operatorname{OSc}} u(\mathrm{x}, \mathrm{t}) \leq M \psi(\mathrm{r})
\end{aligned}
$$

If we using the existence result, then see that there exist a unique solution $v(x, t)$ of problem

$$
\begin{aligned}
& \mathrm{u}_{t}-\operatorname{div}\left(\mathrm{w}(\mathrm{x})|D v|^{p-2} D v\right)=0 \\
& \left.\left.v\right|_{\Gamma(} Q T^{1}\right)=u
\end{aligned}
$$

Let $\mu$ be the nonnegative Riesz measure associated to $v(x, t)$. Note that from existence $\mu$ follows $v(x, t)$ is a supersolution [7]. Let $F=\{(x, t)$ $\left.\in Q_{T}^{1}: v(x, t)=u(x, t)\right\}$. Now prove that the support of $\mu$ is contained in $F \cap E$. For these is sufficient to show that $v(x, t)$ is a weak solution to (2.6) in $Q_{T}^{1} \backslash(F \cup E)$. We already know that (2.6) satisfy in $Q_{T}^{1} \backslash F$ and it therefore remains to show that (2.6) satisfy in $Q_{T=} Q_{T}^{1} \backslash E$. To this aim, we show that if $Q^{*}{ }_{T} \subset Q_{T}^{*}$ is a cylinder and $\alpha € C^{0}\left(Q_{T}^{*}\right)$ is a weak solution to $\alpha_{t}-\left(w(x)|D \alpha|^{P-2} D \alpha\right)$ witk $\alpha=u$ on $\Gamma\left(Q_{T}\right)$, then actually $v$ must coincide with $\alpha$ (x,t)in the $\left(Q_{T}\right)$. Note that such a unique solution $\alpha(x, t)$ exists. We immediately see by the comparison principle that $v \geq$ $\alpha$ in $Q^{*}{ }_{T}$, because $v(x, t)$ is a weak supersolution. To show that $v \leq \alpha$ we 
Citation: Gadjiev TS, Yangaliyeva A, Zulfalieva G (2017) Regularity of Solutions of Degenerate Parabolic Non-linear Equations and Removability of Solutions. J Appl Computat Math 6: 364. doi: 10.4172/2168-9679.1000364

Page 3 of 3

instead argue as follows: since $u(x, t) \leq v(x, t)$, we also have $u(x, t) \leq \alpha(x, t)$ on $\Gamma Q_{T}$ and as $u(x, t)$ solves eqn. (1.1) in $Q_{T}$, the comparison principle holdsu $(\mathrm{x}, \mathrm{t}) \leq \alpha(\mathrm{x}, \mathrm{t})$ in. We thus conclude that $v(x, t) \leq \alpha(x, t)$ on $\Gamma\left(Q^{*}{ }_{T}\right)$ $\cup F$. Therefore $v(x, t)=\alpha(x, t)$ and consequently also eqn. (2.6) yields in $Q^{*}{ }_{T}$. This completes the proof that support of $\mu$ is contained in $F \cap E$.

Later using Theorem 2.1 and a covering argument we can conclude that there exists $C$ depending only on $n, p, v, L, M, \psi(\cdot), Q_{T}^{1}, Q_{T}^{2}$ such that

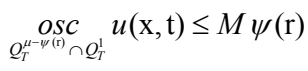

Whenever $\underset{Q_{T}^{1}}{\operatorname{osc}} u(\mathrm{x}, \mathrm{t}) \leq M$. Consider concentric cylinders $\underset{Q_{T}^{\mu-\psi(t)} \cap Q_{T}^{1}}{\operatorname{OSC}} u(\mathrm{x}, \mathrm{t}) \leq M \psi(\mathrm{r})$. In the following we will use the short notation $\bar{Q}_{T}=\mathrm{Q}_{T}^{1} \backslash \mathrm{E}$. Let $\bar{Q}_{T}=\mathrm{Q}_{T}^{1} \backslash \mathrm{E}$ be such 0 and $\phi \equiv 1$ on $Q_{\tau}$. Let $k=\sup v(x, t)$. Using eqn. (2.6) we have

$$
\begin{aligned}
& Q^{\sim} 2 \tau \\
& 0 \leq \mu\left(\bar{Q}_{r}\right) \leq \int_{Q 2 r} \varphi^{p} d \mu= \\
& \int_{Q 2 r}\left[-\left(\varphi^{p}\right)_{t^{v}}+\left(w(x)|D v|^{p-2} D v\right) D \varphi^{p}\right] d x d t \leq \\
& c \int_{Q 2 r} w(x)|D v|^{p-1}|D \varphi| \varphi^{p-1} d x d t+\int_{Q 2 r}\left(\varphi^{p}\right) v d x d t \leq \\
& c\left(\int_{Q 2 r} w(x)|D v|^{p} \varphi^{p} d x d t\right)^{\frac{p-1}{p}}\left(\int_{Q 2 r} w(x)|D \varphi| \varphi^{p} d x d t\right)^{\frac{1}{p}}+ \\
& \int_{Q 2 r}\left(\varphi^{p}\right) v d x d t
\end{aligned}
$$

For the nonnegative weak sub solution $k-v(x, t)$ we see that

$$
c \int_{Q 2 r}\left[\mathrm{w}(\mathrm{x})(k-v)^{p}|D \varphi|^{p-1}+|k-v|^{2}\left|\left(\varphi^{p}\right)_{t}\right|+S^{p} \varphi^{p}\right] d x d t
$$

for some const $c=c(n, p, v, L) \geq 1$. By eqn. (2.7)

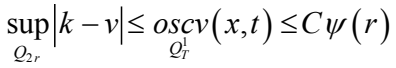

and putting the estimates (2.8) we obtain that

$$
\begin{aligned}
& \mu\left(Q_{T}\right) \leq c\left[\left(\psi^{2}(\tau) \tau^{n}+s^{p} \psi^{2-p}(\tau) \tau^{2-p}\right)\right]^{\frac{p-1}{p}} \cdot\left[|\psi(\tau)|^{2-p} \tau^{n}\right]^{\frac{1}{p}} \\
& c \psi(\tau) \tau^{n} \leq c(1+s)^{p-1} \psi(\tau) \tau^{n} .
\end{aligned}
$$

Here we also used the estimate $|\psi(\tau)|^{2-p} \leq \tau^{2-p}$ for $\tau \leq 1$. Now we consider cylinder $Q_{T}^{3} \subset Q_{T}^{2}$. We will prove that $\mu\left(Q_{T}^{3}\right)=0$. We first note using eqn. (2.9) we have

$$
\mu\left(Q_{\tau}^{w(\tau)}\right) \leq c \tau^{n} \varphi(\tau) .
$$

Whenever $Q_{2 \tau}^{w(2 \tau)} \subset Q_{\tau}^{2}$. Since $H^{\psi(\cdot)}(E)=0$ we obtain for $\varepsilon>0$ and $\delta>0$ given (to be taken smaller that $\operatorname{dist}\left(\Gamma\left(Q_{T}^{3}\right), Q_{T}^{2}\right) / 4$ ), then there exists a countable family

$$
\left\{Q_{\tau_{i}}^{\psi\left(\tau_{i}\right)}\right\}=\left\{Q_{\tau_{i}}^{\psi\left(\tau_{i}\right)}\left(x_{i}, t_{i}\right)\right\}
$$

of cylinders with $0<\tau_{i}<\delta, i=1,2, \ldots$, such that $Q_{2 \tau_{i}}^{\psi\left(2 \tau_{i}\right)} \subset Q_{\tau}^{2}$ and

$$
E \cap Q 3 T \subset[Q \sim \tau \tau i(\tau i) \text { and } \mathrm{X} \tau i n \psi(\tau i)<\varepsilon .
$$

Later using eqn. (2.10) we is obtain

$$
\mu\left[F \cap\left(E \cap Q_{T}^{3}\right)\right] \leq \sum_{i} \mu\left(Q_{\tau_{i}}^{\psi\left(\tau_{i}\right)}\right) \leq \sum_{i} \tau_{i}^{n} \psi\left(\tau_{i}\right)<C . \in
$$

proving that $\mu\left[F \cap\left(E \cap Q_{T}^{3}\right)\right]=0$. The fact that both $Q^{2}{ }_{T}$ and $Q_{T}^{3}$ are arbitrary, we can conclude that $\mu\left(Q_{T}^{1}\right)=0$. Thus $v(x, t)$ is a solution in $Q_{T}^{1}$. Finally applying the above argument with $u(x, t)$ replaced by $-u(x, t)$ we deduce that there exist two solutions $v_{1}(x, t)$ and $v_{2}(x, t)$ i.e., eqn. (2.6) for $v_{1}$ equal to eqn. (2.6) for $v_{2}$. Such that $v_{1}(x, t) \leq u(x, t) \leq v_{2}(x, t)$ and $v_{1}(x, t)=v_{2}(x, t)$ on $\Gamma\left(Q_{T}^{1}\right)$. It follows that $v_{1=} v_{2=} u$. Theorem is proof.

\section{References}

1. Chamillo S, Wheeden RL (1985) Weighted Poincare and Sobolev inequalities Amer J Math 107: 1191-1226.

2. Di Benedetto $E$ (1993) Degenerate parabolic equations. Springer-Verlag.

3. Di Benedetto E, Gianazza U, Vespri V (2008) Intrinsic Harnack Estimates for Non-Negative Solutions of Quasilinear Degenerate Parabolic Equations. Acta Mathematica 200: 181-209.

4. Friedman A (1995) Parabolic variational inequalities in one-space dimension and smoothness of the free boundary. J Func Anal 18: 151-76.

5. Caffarelli LA (1998) The obstacle problem revisited. J Fourier Anal Appl 4 383-402.

6. Serrin J (1964) Local behavior of solutions of quasi-linear equations. Acta Mathe-matica 3: 247-302.

7. Heinonen J, Kilpelainen $T$ (1998) A superharmonic functions and supersolutions of degenerate elliptic equations. Arkiv Matematik 26: 87-105.

8. Kilpelainen T, Zhong X (2002) Removable sets for continuous solutions of quasi-linear elliptic equations. Proceedings of the American Mathematical Society 130: 1681-1688.

9. Gadjiev TS, Sadigova NR, Rasulov RA (2011) Removable singularities of solutions of degenerate nonlinear elliptic equations on the boundary of a domain. Nonlinear Analysis: Theory, Methods and Applications 74: 5566-5571.

10. Gadjiev T, Bayramova N (2014) The removability of compact of solutions in classes bounded functions. Ukrayne Mathematics Journal 8: 38-44.

11. Gadjiev T, Aliev O (2013) On Removable Sets of Solutions of Neuman Problem for Quasilinear Elliptic Equations of Divergent Form. Applied Mathematics 4: 290-298

12. Gadjiev T (2013) On Removable Sets for Generated Elliptic Equations. British Journal of Mathematics and Computer Science 3: 290-298. 